\title{
Upper bound and shareability of quantum discord based on entropic uncertainty relations
}

\author{
Ming-Liang Hu用 and Heng Fan讨 \\ ${ }^{1}$ School of Science, Xi'an University of Posts and Telecommunications, Xi'an 710121, China \\ ${ }^{2}$ Beijing National Laboratory for Condensed Matter Physics, \\ Institute of Physics, Chinese Academy of Sciences, Beijing 100190, China
}

\begin{abstract}
By using the quantum-memory-assisted entropic uncertainty relation (EUR), we derive a computable tight upper bound for quantum discord, which applies to an arbitrary bipartite state. Detailed examples show that this upper bound is tighter than other known bounds in a wide regime. Furthermore, we show that for any tripartite pure state, the quantum-memory-assisted EUR imposes a constraint on the shareability of quantum correlations among the constituent parties. This conclusion amends the well accepted result that quantum discord is not monogamous.

PACS numbers: 03.67.Mn, 03.65.Ta, 03.65.Yz
\end{abstract}

Quantum correlations are of special importance in quantum information processing, such as in the deterministic quantum computation with one qubit [1], and other related quantum protocols [2-4]. Among different measures of quantum correlation, quantum discord (QD) [5] has been attracting particular attention. Various aspects of QD , e.g., the role it played in identifying quantum phase transition [6], its local creativity [7], and operational interpretation [8], have been explored. Its peculiar behaviors in evolution under noisy environments [9, 10] have also been investigated.

Despite the significance, the value of QD is notoriously difficult to calculate due to the optimization procedure involved. Analytical results are known only for certain special classes of states [11-13]. Particularly, it has been proved that it is impossible to obtain a closed expression for $\mathrm{QD}$, even for general states of two qubits [14]. This fact makes it desirable to obtain some computable bounds for QD, and several attempts have been devoted to this issue in the past few years [15-18].

In this work, we reexamined the above issue from some alternative perspectives. We noted that the quantum correlation plays a deterministic role in improving the prediction precision of an imaginary "uncertainty game" [19], which has been further explored in several recently published papers [20]. Particularly, a connection between entanglement and measurement uncertainty was established in a very recent work [21]. Here, instead of concentrating on the role that QD played in tightening the lower bound of the new entropic uncertainty relation (EUR) [22], we reversely consider how this EUR constrains the magnitude of QD. Our study shows that from the uncertainty principle represented as the EUR, one can derive certain improved upper bounds for QD. These bounds are tighter in a wide regime than those obtained in the literature [15, 16].

Another issue we will study is how the EUR affects shareability of quantum correlations among different subsystems. It is well known that QD does not satisfy the monogamy relation which is considered a fundamental property concerning the resource shareability among multi-parties [23]. Naturally,

\footnotetext{
*Electronic address: mingliang0301@163.com
}

†Electronic address: hfan@iphy.ac.cn a question arises as to whether there exists any constraint on the shareability of QD. We find that the EUR sets a fundamental limit on the shareability of QD for all the tripartite pure states. This can be considered as an amendment to the fact that $\mathrm{QD}$ violates the monogamy condition.

Let us first recall the definition of QD, which is based on the partition of the total correlations in a state $\rho_{A B}$, measured by the quantum mutual information $I\left(\rho_{A B}\right)=S\left(\rho_{A}\right)+S\left(\rho_{B}\right)-$ $S\left(\rho_{A B}\right)$, into two different parts, i.e., the classical part and the quantum part. The classical part $J_{A}\left(\rho_{A B}\right)$, also known as the classical correlation [5], is defined as

$$
J_{A}\left(\rho_{A B}\right)=S\left(\rho_{B}\right)-\min _{\left\{E_{k}^{A}\right\}} S\left(B \mid\left\{E_{k}^{A}\right\}\right),
$$

where $S\left(\rho_{B}\right)=-\operatorname{Tr}\left(\rho_{B} \log _{2} \rho_{B}\right)$ denotes the von Neumann entropy of the reduced density operator $\rho_{B}=\operatorname{Tr}_{A} \rho_{A B}$, and $S\left(B \mid\left\{E_{k}^{A}\right\}\right)=\sum_{k} p_{k} S\left(\rho_{B \mid k}\right)$ is the averaged conditional von Neumann entropy of the nonselective postmeasurement state $\rho_{B \mid k}=\operatorname{Tr}_{A}\left(E_{k}^{A} \rho_{A B}\right) / p_{k}$ after the positive operator valued measure (POVM) on party $A$, with $p_{k}=\operatorname{Tr}\left(E_{k}^{A} \rho_{A B}\right)$.

The quantum part $D_{A}\left(\rho_{A B}\right)$, which is QD under our consideration, is then obtained by subtracting $J_{A}\left(\rho_{A B}\right)$ from $I\left(\rho_{A B}\right)$ [5], namely,

$$
D_{A}\left(\rho_{A B}\right)=\min _{\left\{E_{k}^{A}\right\}} S\left(B \mid\left\{E_{k}^{A}\right\}\right)-S(B \mid A),
$$

where $S(B \mid A)=S\left(\rho_{A B}\right)-S\left(\rho_{A}\right)$ denotes the conditional von Neumann entropy of $\rho_{A B}$. For general $\rho_{A B}$, a tight upper bound for QD is proven to be [15]

$$
D_{A}\left(\rho_{A B}\right) \leq S\left(\rho_{A}\right),
$$

with equality holding if and only if the complex Hilbert space of subsystem $B$ can be decomposed as $\mathcal{H}_{B}=\mathcal{H}_{B^{L}} \otimes \mathcal{H}_{B^{R}}$ such that $\rho_{A B}=|\psi\rangle_{A B^{L}}\langle\psi| \otimes \rho_{B^{R}}$ [16].

The quantum-memory-assisted EUR was initially conjectured by Renes and Boileau [24] and then proven by Berta et al. [19]. It reads

$$
S(Q \mid B)+S(R \mid B) \geq \log _{2} \frac{1}{c}+S(A \mid B),
$$

where $S(X \mid B)$ is the conditional entropy of the postmeasurement state $\rho_{X B}=\sum_{k}\left(\left|\psi_{k}^{X}\right\rangle\left\langle\psi_{k}^{X}\right| \otimes \mathbb{I}\right) \rho_{A B}\left(\left|\psi_{k}^{X}\right\rangle\left\langle\psi_{k}^{X}\right| \otimes \mathbb{I}\right)$, 
with $\left|\psi_{k}^{X}\right\rangle$ being the eigenvectors of $X=\{Q, R\}$. Moreover, $c$ in Eq. (4) quantifies the incompatibility of the observables $Q$ and $R$. It is defined as $c=\max _{k, l}\left|\left\langle\psi_{k}^{Q} \mid \psi_{l}^{R}\right\rangle\right|^{2}$. Here, the subsystem $B$ is called a quantum memory, as it stores information which can be used by one player of the uncertainty game to infer the measurement outcome of his counterpart [19].

Experimentally, the EUR of Eq. (4) has been tested in systems of photon pairs [25], and is proposed for testing in nitrogen-vacancy (NV) center in diamond [26]. Theoretically, a tighter lower bound of measurement uncertainty than that presented in the right-hand side (RHS) of Eq. (4) is obtained in a recent work [22]. By incorporating the discrepancy between QD and the classical correlation into account, the following inequality is proven:

$$
S(Q \mid B)+S(R \mid B) \geq \log _{2} \frac{1}{c}+S(A \mid B)+\max \{0,-\Delta\},
$$

where $\Delta=J_{A}\left(\rho_{A B}\right)-D_{A}\left(\rho_{A B}\right)$ characterizes the imbalance between the classical correlation and QD [27]. Therefore, the lower bound of Berta et al. [Eq. (4)] is tightened whenever $\Delta<0$, i.e., when the quantum correlation in the joint system of the quantum memory and the measured particle exceeds the classical correlation that exists in the same system.

We remark here that the correlation discrepancy $\Delta$ equals $I\left(\rho_{B C}\right)-2 E_{f}\left(\rho_{B C}\right)$ when one takes the purified state $|\Psi\rangle_{A B C}$ for $\rho_{A B}$ into consideration [27], where $E_{f}\left(\rho_{B C}\right)=$ $\min \sum_{i} p_{i} S\left(\operatorname{Tr}_{C}\left|\psi_{i}\right\rangle_{B C}\left\langle\psi_{i}\right|\right)$ represents the entanglement of formation (EoF) [28] for state $\rho_{B C}=\operatorname{Tr}_{A}\left(|\Psi\rangle_{A B C}\langle\Psi|\right)$, and the minimum is taken over all the pure-state decompositions $\rho_{B C}=\sum_{i} p_{i}\left|\psi_{i}\right\rangle_{B C}\left\langle\psi_{i}\right|$. Meanwhile, $I\left(\rho_{B C}\right)-2 E_{f}\left(\rho_{B C}\right)$ is also found to be equal to $\tau_{D}=D_{A}\left(\rho_{A: B C}\right)-D_{A}\left(\rho_{A B}\right)-$ $D_{A}\left(\rho_{A C}\right)$ [29]. $\tau_{D}$ is the discord monogamy score introduced in [30]. Therefore, we have $\Delta=\tau_{D}$, which indicates that the lower bound of the EUR in Eq. (4) is improved whenever the purification $|\Psi\rangle_{A B C}$ for $\rho_{A B}$ violates the monogamy inequality $D_{A}\left(\rho_{A B}\right)+D_{A}\left(\rho_{A C}\right) \leq D_{A}\left(\rho_{A: B C}\right)$, i.e., $\Delta<0$ whenever $|\Psi\rangle_{A B C}$ is not monogamous.

With the above preliminaries, we now show applications of the EUR (5) in deriving improved upper bounds on QD. To this end, and for the purpose of showing figures of merit using this method, we first introduce a slightly stronger upper bound of QD than that presented in Eq. (3), which is given by

$$
D_{A}\left(\rho_{A B}\right) \leq \min \left\{S\left(\rho_{A}\right), S\left(\rho_{A}\right)-S(A \mid B)\right\} .
$$

This upper bound tightens that presented in Eq. (3) for all $\rho_{A B}$ with positive conditional entropy $S(A \mid B)\left[D_{A}\left(\rho_{A B}\right) \leq\right.$ $S\left(\rho_{A}\right)-S(A \mid B)=I\left(\rho_{A B}\right)$ holds obviously true by its definition]. This occurs for several natural bipartite states; see, for example, the dashed lines in Fig. 1 1 obtained by Eq. (6) for the $d \otimes d$ pseudopure state [13]

$$
\rho_{\mathrm{PP}}=\frac{1-r}{d^{2}-1} \mathbb{I}+\frac{r d^{2}-1}{d^{2}-1}|\psi\rangle\langle\psi|,
$$

where $|\psi\rangle=\sum_{i=1}^{d} u_{i}|i i\rangle$, with $\sum_{i=1}^{d} u_{i}^{2}=1$. For both plots displayed in Fig. 1, the dashed lines before the sudden change points $r_{S C}$ denoted by the stars correspond to the upper bounds of $D_{A}\left(\rho_{A B}\right)$ given by $S\left(\rho_{A}\right)-S(A \mid B)$, while after the points $r_{S C}$, they are given by the original bound $S\left(\rho_{A}\right)$.
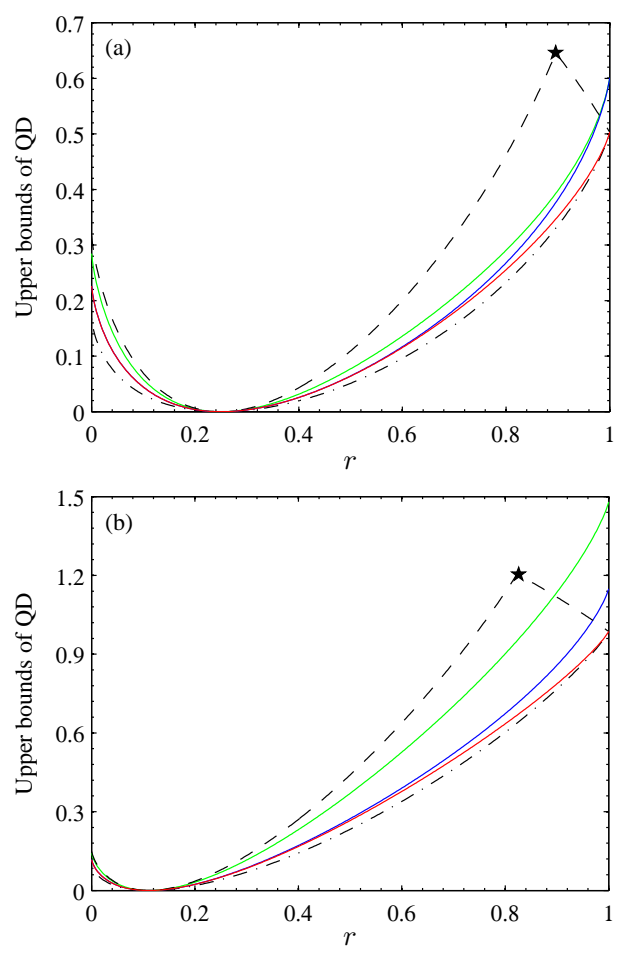

FIG. 1: (Color online) Upper bounds of QD for $\rho_{\mathrm{PP}}$ of Eq. (7) with (a) $d=2, u_{1}=2 \sqrt{2} / 3, u_{2}=1 / 3$ and (b) $d=3, u_{1}=\sqrt{7} / 3$, $u_{2,3}=1 / 3$. The dash-dotted and the dashed lines are the exact results of QD and its upper bounds given by Eq. (6) [the stars denote critical points after which $S\left(\rho_{A}\right)$ in Eq. 6) dominates], while the solid red, blue, and green lines (from bottom to top) are those given by $\Lambda_{\alpha}$ [Eqs. (8) and [10] with $\alpha=\mathrm{T}, \mathrm{M}$, and F, respectively.

Due to the high symmetry of $\rho_{\mathrm{PP}}$, analytical results of the QD can also be obtained [13] and they are denoted by the dashdotted lines illustrated in Fig. 1 .

Based on the result of Eq. (6), we now present our improved upper bound to QD via the following theorem.

Theorem 1. For any bipartite state $\rho_{A B}$, the QD satisfies

$$
D_{A}\left(\rho_{A B}\right) \leq \min \left\{S\left(\rho_{A}\right), I\left(\rho_{A B}\right), \Lambda_{\mathrm{T}}\right\},
$$

where $\Lambda_{\mathrm{T}}=\left[\delta_{\mathrm{T}}+I\left(\rho_{A B}\right)\right] / 2$, and

$$
\delta_{\mathrm{T}}=S(Q \mid B)+S(R \mid B)-\log _{2} \frac{1}{c}-S(A \mid B),
$$

which characterizes the discrepancy between uncertainty of the measurement outcomes of $Q$ and $R$ (inferred from projective measurement on $A$ and quantum state tomography on $B$, known as the tomographic estimate in [25]) and its lower bound depicted on the RHS of Eq. (4).

Proof. Due to Eq. (6), it suffices to prove that (i) the inequality $D_{A}\left(\rho_{A B}\right) \leq \Lambda_{\mathrm{T}}$, and (ii) it is possible for $\Lambda_{\mathrm{T}}$ to be smaller than or equal to $S\left(\rho_{A}\right)$ and $I\left(\rho_{A B}\right)$.

The first one, that is, $D_{A}\left(\rho_{A B}\right) \leq \Lambda_{\mathrm{T}}$, can be shown to be true by reexpressing $\Delta$ in Eq. (5) as $I\left(\rho_{A B}\right)-2 D_{A}\left(\rho_{A B}\right)$, which gives immediately $\delta_{\mathrm{T}} \geq 2 D_{A}\left(\rho_{A B}\right)-I\left(\rho_{A B}\right)$, and therefore $D_{A}\left(\rho_{A B}\right) \leq \Lambda_{\mathrm{T}}$. 
The second one can be proven by taking the minimum uncertainty states of Berta et al. [e.g., the Greenberger-HorneZeilinger state $(|000\rangle+|111\rangle) / \sqrt{2}$; refer to [31] for more details about this kind of state] as an example, which correspond to $\delta_{\mathrm{T}}=0$. Therefore, the requirement (ii) reduces to $I\left(\rho_{A B}\right) / 2 \leq S\left(\rho_{A}\right)$ and $I\left(\rho_{A B}\right) / 2 \leq I\left(\rho_{A B}\right)$. The former one can always be satisfied due to the Araki-Lieb inequality $\left|S\left(\rho_{A}\right)-S\left(\rho_{B}\right)\right| \leq S\left(\rho_{A B}\right)$ [32] and the latter one is obvious. This completes the proof.

One can make the upper bound $\Lambda_{\mathrm{T}}$ better by choosing appropriate observables. Particularly, when $Q$ and $R$ are complementary such that $\log _{2}(1 / c)=\log _{2} d_{A}$, with $d_{A}$ being the dimension of $\mathcal{H}_{A}$, the upper bound $\Lambda_{\mathrm{T}}$ of $D_{A}\left(\rho_{A B}\right)$ in Eq. (8) is saturated for the isotropic state of arbitrary dimensions [33], i.e., for all $\rho_{\mathrm{PP}}$ of Eq. (7) with $u_{i}=1 / \sqrt{d}$. Thus the upper bound $\Lambda_{\mathrm{T}}$ we obtained is tight.

For general $\rho_{\mathrm{PP}}$, from the solid red lines shown in Fig. 1 which are obtained by choosing the observables $Q$ and $R$ such that $\log _{2}(1 / c)=\log _{2} d$ [note that for $\rho_{\mathrm{PP}}$ in Eq. (7), $d_{A}=$ $\left.d_{B}=d\right]$, one can see obviously that the upper bound $\Lambda_{\mathrm{T}}$ tightens that given by Eq. (6), and for the special cases of $r=1 / d^{2}$ [with $D_{A}\left(\rho_{A B}\right)=0$ ] and $r=1$ [with $D_{A}\left(\rho_{A B}\right)=$ $\left.-\sum_{i=1}^{d} u_{i}^{2} \log _{2} u_{i}^{2}\right]$, the upper bound $\Lambda_{\mathrm{T}}$ is saturated.

Moreover, by using the facts that projective measurements increase entropy [32] and Fano's inequality [19, 25], one can obtain two slightly weaker bounds for QD as follows:

$$
D_{A}\left(\rho_{A B}\right) \leq \min \left\{S\left(\rho_{A}\right), I\left(\rho_{A B}\right), \Lambda_{\alpha}\right\},
$$

where $\Lambda_{\alpha}=\left[\delta_{\alpha}+I\left(\rho_{A B}\right)\right] / 2$, with $\alpha=\{\mathrm{M}, \mathrm{F}\}$. Similarly, $\delta_{\mathrm{M}}$ and $\delta_{\mathrm{F}}$ can be obtained directly by replacing the first two terms on the RHS of Eq. (9) with $S(Q \mid Q)+S(R \mid R)$ and $h\left(p_{Q}\right)+h\left(p_{R}\right)+\left(p_{Q}+p_{R}\right) \log _{2}\left(d_{A}-1\right)$, respectively. Here, $S(X \mid X)(X=Q, R)$ denotes the conditional von Neumann entropy of the postmeasurement state $\rho_{X X}$ obtained via twoside projective measurements on $\rho_{A B}$, and $h\left(p_{X}\right)$ is the binary entropy of the probability distribution $p_{X}$ corresponding to different outcomes of $X$ on $A$ and $X$ on $B$.

The upper bounds given in Eq. (10) are, in general, weaker than that of Eq. (8) in that $\Lambda_{\mathrm{M}, \mathrm{F}} \geq \Lambda_{\mathrm{T}}$, but may be favored for their ease of experimental accessibility [25]. Particularly, they may still be tighter than that given by Eq. (6) under certain circumstances. See, for example, the solid blue (given by $\Lambda_{\mathrm{M}}$ ) and solid green (given by $\Lambda_{\mathrm{F}}$ ) lines displayed in Fig. 1 for the pseudopure states of Eq. (7), which are nearly overlapped during the small $r$ regions. Clearly, both of the bounds described by $\Lambda_{\mathrm{M}}$ and $\Lambda_{\mathrm{F}}$ are tighter than that given by Eq. (6) in most intervals of the mixing parameter $r$.

At this stage, one may wonder what the other implications would be of the tightened EUR in Eq. (5). Here, we show that it also implies a constraint on the shareability of QD among different parties of a composite system.

Theorem 2. For any tripartite state $\rho_{A B C}$ with $S\left(\rho_{A}\right)=$ $-S(A \mid B C)$, we have

$$
D_{A}\left(\rho_{A B}\right)+D_{A}\left(\rho_{A C}\right) \leq D_{A}\left(\rho_{A: B C}\right)+\delta_{\mathrm{T}} .
$$

Proof. First, Eq. (5) means $\delta_{\mathrm{T}} \geq D_{A}\left(\rho_{A B}\right)-J_{A}\left(\rho_{A B}\right)$. This, together with the inequality $D_{A}\left(\rho_{A C}\right)+J_{A}\left(\rho_{A B}\right) \leq$
$S\left(\rho_{A}\right)$ which is applicable for arbitrary $\rho_{A B C}$ [34], results in

$$
D_{A}\left(\rho_{A B}\right)+D_{A}\left(\rho_{A C}\right) \leq S\left(\rho_{A}\right)+\delta_{\mathrm{T}} .
$$

Thus, Eq. (11) holds obviously for all the tripartite pure states $|\Psi\rangle_{A B C}$ because we always have $D_{A}\left(\rho_{A: B C}\right)=S\left(\rho_{A}\right)$, and $S\left(\rho_{A}\right)=-S(A \mid B C)$. Moreover, we know from Ref. [16] that even for mixed $\rho_{A B C}, S\left(\rho_{A}\right)=-S(A \mid B C)$ if and only if there exists a factorization $\mathcal{H}_{B C}=\mathcal{H}_{(B C)^{L}} \otimes \mathcal{H}_{(B C)^{R}}$ for the Hilbert space $\mathcal{H}_{B C}$ such that $\rho_{A B C}=|\psi\rangle_{A(B C)^{L}}\langle\psi| \otimes$ $\rho_{(B C)^{R}}$, and therefore $D_{A}\left(\rho_{A: B C}\right)=D_{A}\left(|\psi\rangle_{A(B C)^{L}}\right)=$ $S\left(\rho_{A}\right)$.

The inequality (11) is a released version of the monogamy relation of QD [23]. It applies for all tripartite pure states and to extended classes of mixed states. As $\delta_{\mathrm{T}}$ is non-negative due to Eq. (4), the inequality (11) implies immediately that even if QD may violate the monogamy inequality, the different subsystems of $A B C$ still cannot be freely correlated. That is, subsystem $A$ cannot share an unlimited amount of quantum correlations individually with both $B$ and $C$, as the summation is limited by $D_{A}\left(\rho_{A: B C}\right)+\delta_{\mathrm{T}}$. Therefore, we see that although the monogamy inequality of QD may be violated, there exists a limitation for QD shareability.

Moreover, as a corollary, we emphasize here that Eq. (11) also yields a sufficient condition for the monogamy of QD in a class of tripartite states $\rho_{A B C}$ with $S\left(\rho_{A}\right)=-S(A \mid B C)$. That is, there exist measurement operators $Q$ and $R$ such that the discrepancy $\delta_{\mathrm{T}}$ defined in Eq. (9) vanishes, i.e., $\delta_{\mathrm{T}}=$ 0 . This occurs, for instance, for the reduced $\rho_{A B}$ being the minimum uncertainty state of Berta et al. [31], namely, the bipartite states $\rho_{A B}=\operatorname{Tr}_{C}\left(|\Psi\rangle_{A B C}\langle\Psi|\right)$ saturate the lower bound of Eq. (4).

Finally, it is worthwhile to note that the original definition of QD introduced by Ollivier and Zurek [5] is measurement dependent, thus there are two possible lines for studying the monogamous character of QD. The case we discussed in Theorem 2 corresponds to that with the measurements being performed on the same subsystem $A$. When we go forward along another line for which the measurements were performed on different subsystems (some discussions along this line can be found in [23, 35]), we can also establish a connection between the EUR and shareability of QD.

Theorem 3. For any tripartite pure state $|\Psi\rangle_{A B C}$, we have

$$
D_{B}\left(\rho_{A B}\right)+D_{C}\left(\rho_{A C}\right) \leq D_{B C}\left(\rho_{A: B C}\right)+\bar{\delta}_{\mathrm{T}}
$$

where $\bar{\delta}_{\mathrm{T}}=\left[\delta_{\mathrm{T}}^{(B A)}+\delta_{\mathrm{T}}^{(C A)}\right] / 2$, with

$$
\begin{aligned}
\delta_{\mathrm{T}}^{(B A)} & =S\left(Q_{B} \mid A\right)+S\left(R_{B} \mid A\right)-\log _{2} \frac{1}{c}-S(B \mid A), \\
\delta_{\mathrm{T}}^{(C A)} & =S\left(Q_{C} \mid A\right)+S\left(R_{C} \mid A\right)-\log _{2} \frac{1}{c}-S(C \mid A) .
\end{aligned}
$$

Proof. By making the substitutions $A \rightarrow X(X=B$ or $C)$ and $B \rightarrow A$ to the EUR in Eq. (5), we obtain

$$
D_{X}\left(\rho_{A X}\right) \leq \frac{1}{2}\left[\delta_{\mathrm{T}}^{(X A)}+S\left(\rho_{X}\right)-S(X \mid A)\right],
$$


for arbitrary $\rho_{A B C}$. Consequently,

$$
\begin{aligned}
D_{B}\left(\rho_{A B}\right)+D_{C}\left(\rho_{A C}\right) \leq & S\left(\rho_{A}\right)+\bar{\delta}_{\mathrm{T}} \\
& -\frac{1}{2}[S(A \mid B)+S(A \mid C)] \\
\leq & S\left(\rho_{A}\right)+\bar{\delta}_{\mathrm{T}},
\end{aligned}
$$

where the second inequality is due to the strong subadditivity of the von Neumann entropy [32]. Then, Eq. (13) is obviously true for any $|\Psi\rangle_{A B C}$ because for pure states we always have $D_{B C}\left(\rho_{A: B C}\right)=D_{A}\left(\rho_{A: B C}\right)=S\left(\rho_{A}\right)$.

This theorem also implies that if there exist observables $Q$ and $R$ giving nullity of $\bar{\delta}_{\mathrm{T}}$, then the state $|\Psi\rangle_{A B C}$ will obey the monogamy of discord condition $D_{B}\left(\rho_{A B}\right)+D_{C}\left(\rho_{A C}\right) \leq$ $D_{B C}\left(\rho_{A: B C}\right)$. It shows again the power of the EUR for exploring monogamy properties of discord.

In summary, we have shown applications of a generalized EUR [22] in obtaining an improved tight upper bound for QD. This bound applies to bipartite states of arbitrary dimensions and tightens that given in the literature [15, 16]. In addition, we have also shown applications of the EUR in identifying an inequality which constrains the shareability of QD between different parties of a composite system. More specifically, we showed that even if QD may not respect the monogamy relation, the quantum correlations still cannot be freely shared. As an amendment to the violation of monogamy relation for QD, a released monogamylike relation is still satisfied for all tripartite pure states. We hope that these results may provide useful insights as to what role quantum correlations play in the fundamental theory of the uncertainty principle, as well as to how the uncertainty principle, particularly those of the entropic forms, imposes constraints on the strength and distributions of quantum correlations.

This work was supported by NSFC (11205121, 10974247, 11175248), the "973" program (2010CB922904), NSF of Shaanxi Province (2010JM1011), and the Scientific Research Program of the Education Department of Shaanxi Provincial Government (12JK0986).
[1] A. Datta, A. Shaji, and C. M. Caves, Phys. Rev. Lett. 100, 050502 (2008); B. P. Lanyon, M. Barbieri, M. P. Almeida, and A. G. White, ibid. 101, 200501 (2008); B. Dakić, V. Vedral, and Č. Brukner, ibid. 105, 190502 (2010).

[2] B. Dakić et al., Nat. Phys. 8, 666 (2012).

[3] M. Gu et al., Nat. Phys. 8, 671 (2012).

[4] K. Modi, A. Brodutch, H. Cable, T. Paterek, and V. Vedral, Rev. Mod. Phys. 84, 1655 (2012).

[5] H. Ollivier and W. H. Zurek, Phys. Rev. Lett. 88, 017901 (2001); L. Henderson and V. Vedral, J. Phys. A 34, 6899 (2001).

[6] R. Dillenschneider, Phys. Rev. B 78, 224413 (2008); M. S. Sarandy, Phys. Rev. A 80, 022108 (2009); T. Werlang, C. Trippe, G. A. P. Ribeiro, and G. Rigolin, Phys. Rev. Lett. 105, 095702 (2010).

[7] A. Streltsov, H. Kampermann, and D. Bruß, Phys. Rev. Lett. 107, 170502 (2011); X. Hu, H. Fan, D. L. Zhou, and W.-M. Liu, Phys. Rev. A 85, 032102 (2012); M. Gessner, E.-M. Laine, H.-P. Breuer, and J. Piilo, ibid. 85, 052122 (2012); T. Abad, V. Karimipour, and L. Memarzadeh, ibid. 86, 062316 (2012).

[8] V. Madhok and A. Datta, Phys. Rev. A 83, 032323 (2011); D. Cavalcanti, L. Aolita, S. Boixo, K. Modi, M. Piani, and A. Winter, ibid. 83, 032324 (2011).

[9] L. Mazzola, J. Piilo, and S. Maniscalco, Phys. Rev. Lett. 104, 200401 (2010).

[10] F. F. Fanchini, T. Werlang, C. A. Brasil, L. G. E. Arruda, and A. O. Caldeira, Phys. Rev. A 81, 052107 (2010); J. Maziero, L. C. Céleri, R. M. Serra, and V. Vedral, ibid. 80, 044102 (2009); T. Werlang, S. Souza, F. F. Fanchini, and C. J. Villas Boas, ibid. 80, 024103 (2009).

[11] S. Luo, Phys. Rev. A 77, 042303 (2008); M. Ali, A. R. P. Rau, and G. Alber, ibid. 81, 042105 (2010); Q. Chen, C. Zhang, S. Yu, X. X. Yi, and C. H. Oh, ibid. 84, 042313 (2011).

[12] L.-X. Cen, X.-Q. Li, J. Shao, and Y. J. Yan, Phys. Rev. A 83, 054101 (2011).

[13] E. Chitambar, Phys. Rev. A 86, 032110 (2012).

[14] D. Girolami and G. Adesso, Phys. Rev. A 83, 052108 (2011).

[15] A. Datta, arXiv:0807.4490, S. Luo, S. Fu, and N. Li, Phys. Rev.
A 82, 052122 (2010).

[16] Z. Xi, X.-M. Lu, X. Wang, and Y. Li, Phys. Rev. A 85, 032109 (2012).

[17] S. Yu, C. Zhang, Q. Chen, and C. H. Oh, arXiv:1102.1301.

[18] C. Zhang, S. Yu, Q. Chen, and C. H. Oh, Phys. Rev. A 84, 052112 (2011).

[19] M. Berta, M. Christandl, R. Colbeck, J. M. Renes, and R. Renner, Nat. Phys. 6, 659 (2010).

[20] M. L. Hu and H. Fan, Phys. Rev. A 86, 032338 (2012); M. L. $\mathrm{Hu}$ and H. Fan, ibid. 87, 022314 (2013); Z. Y. Xu, W. L. Yang, and M. Feng, ibid. 86, 012113 (2012).

[21] M. Berta, P. J. Coles, and S. Wehner, arXiv:1302.5902

[22] A. K. Pati, M. M. Wilde, A. R. Usha Devi, A. K. Rajagopal, and Sudha, Phys. Rev. A 86, 042105 (2012).

[23] A. Streltsov, G. Adesso, M. Piani, and D. Bruß, Phys. Rev. Lett. 109, 050503 (2012); G. L. Giorgi, Phys. Rev. A 84, 054301 (2011); R. Prabhu, A. K. Pati, A. Sen(De), and U. Sen, ibid. 85, 040102 (2012); H. C. Braga, C. C. Rulli, T. R. de Oliveira, and M. S. Sarandy, ibid. 86, 062106 (2012).

[24] J. M. Renes and J. C. Boileau, Phys. Rev. Lett. 103, 020402 (2009).

[25] C.-F. Li, J.-S. Xu, X.-Y. Xu, K. Li, and G.-C. Guo, Nat. Phys. 7, 752 (2011); R. Prevedel, D. R. Hamel, R. Colbeck, K. Fisher, and K. J. Resch, ibid. 7, 757 (2011).

[26] Z. Y. Xu, S. Q. Zhu, and W. L. Yang, Appl. Phys. Lett. 101, 244105 (2012).

[27] F. F. Fanchini, M. C. de Oliveira, L. K. Castelano, and M. F. Cornelio, Phys. Rev. A 87, 032317 (2013).

[28] C. H. Bennett, D. P. DiVincenzo, J. A. Smolin, and W. K. Wootters, Phys. Rev. A 54, 3824 (1996); W. K. Wootters, Phys. Rev. Lett. 80, 2245 (1998).

[29] X. J. Ren and H. Fan, Quantum Inf. Comput. 13, 0469 (2013).

[30] M. N. Bera, R. Prabhu, A. Sen(De), and U. Sen, Phys. Rev. A 86, 012319 (2012).

[31] P. J. Coles, R. Colbeck, L. Yu, and M. Zwolak, Phys. Rev. Lett. 108, 210405 (2012); P. J. Coles, L. Yu, and M. Zwolak, arXiv:1105.4865 
[32] M. A. Nielsen and I. L. Chuang, Quantum Computation and Quantum Information (Cambridge University Press, Cambridge, UK, 2000).

[33] B. M. Terhal and K. G. H. Vollbrecht, Phys. Rev. Lett. 85, 2625 (2000).

[34] M. Koashi and A. Winter, Phys. Rev. A 69, 022309 (2004).
[35] F. F. Fanchini, M. F. Cornelio, M. C. de Oliveira, and A. O. Caldeira, Phys. Rev. A 84, 012313 (2011); F. F. Fanchini, L. K. Castelano, M. F. Cornelio, and M. C. de Oliveira, New J. Phys. 14, 013027 (2012). 